\title{
A Clinical Prediction Model for Long-Term Functional Outcome after Traumatic Spinal Cord Injury Based on Acute Clinical and Imaging Factors
}

\author{
Jefferson R. Wilson, ${ }^{1}$ Robert G. Grossman, ${ }^{2}$ Ralph F. Frankowski, ${ }^{3}$ Alexander Kiss, ${ }^{4}$ Aileen M. Davis, \\ Abhaya V. Kulkarni,, James S. Harrop, ${ }^{1,5}$ Bizhan Aarabi, ${ }^{7}$ Alexander Vaccaro, ${ }^{6}$ Charles H. Tator, \\ Marcel Dvorak, ${ }^{8}$ Christopher I. Shaffrey, ${ }^{9}$ Susan Harkema, ${ }^{10}$ James D. Guest, ${ }^{11}$ and Michael G. Fehlings ${ }^{1}$
}

\begin{abstract}
To improve clinicians' ability to predict outcome after spinal cord injury (SCI) and to help classify patients within clinical trials, we have created a novel prediction model relating acute clinical and imaging information to functional outcome at 1 year. Data were obtained from two large prospective SCI datasets. Functional independence measure (FIM) motor score at 1 year follow-up was the primary outcome, and functional independence (score $\geq 6$ for each FIM motor item) was the secondary outcome. A linear regression model was created with the primary outcome modeled relative to clinical and imaging predictors obtained within 3 days of injury. A logistic model was then created using the dichotomized secondary outcome and the same predictor variables. Model validation was performed using a bootstrap resampling procedure. Of 729 patients, 376 met the inclusion criteria. The mean FIM motor score at 1 year was $62.9( \pm 28.6)$. Better functional status was predicted by less severe initial American Spinal Injury Association (ASIA) Impairment Scale grade, and by an ASIA motor score $>50$ at admission. In contrast, older age and magnetic resonance imaging (MRI) signal characteristics consistent with spinal cord edema or hemorrhage predicted worse functional outcome. The linear model predicting FIM motor score demonstrated an R-square of 0.52 in the original dataset, and 0.52 (95\% CI $0.52,0.53$ ) across the 200 bootstraps. Functional independence was achieved by 148 patients $(39.4 \%)$. For the logistic model, the area under the curve was 0.93 in the original dataset, and $0.92(95 \%$ CI $0.92,0.93)$ across the bootstraps, indicating excellent predictive discrimination. These models will have important clinical impact to guide decision making and to counsel patients and families.
\end{abstract}

Key words: clinical prediction model; functional outcome; spinal cord injury

\section{Introduction}

$\mathbf{S}_{\mathrm{s}}^{\mathrm{r}}$ PINAL CORD INJURY (SCI) is a devastating and debilitating condition that affects all regions of the world (Wyndaele and Wyndaele, 2006). Over one million people are living with
SCI in the United States at present, with annual costs for the acute treatment and chronic care of these patients totaling four billion dollars (Christopherand Dana Reeve Foundation, 2011). In spite of the immense impact of SCI at a personal and societal level, comprehensive treatment strategies aimed

\footnotetext{
${ }^{1}$ Department of Surgery, Division of Neurosurgery and Spinal Program, ${ }^{4}$ Department of Research Design and Biostatistics, Sunnybrook Research Institute, and ${ }^{5}$ Department of Health Policy, Management and Evaluation, University of Toronto, Toronto Western Hospital, Toronto, Ontario, Canada.

${ }^{2}$ Department of Neurosurgery, The Methodist Hospital, Houston, Texas.

${ }^{3}$ School of Public Health, University of Texas, Houston, Texas.

${ }^{6}$ Department of Neurosurgery and Orthopedic Surgery, Division of Spinal Disorders, Thomas Jefferson University, Philadelphia, Pennsylvania.

${ }^{7}$ Department of Neurosurgery, University of Maryland, Baltimore, Maryland.

${ }^{8}$ Department of Orthopedic Surgery, University of British Columbia, Vancouver, British Columbia, Canada.

${ }^{9}$ Departments of Neurosurgery and Orthopedic Surgery, University of Virginia, Charlottesville, Virginia.

${ }^{10}$ Department of Neurosurgery, University of Kentucky, Louisville, Kentucky.

${ }^{11}$ Department of Neurosurgery and Miami Project to Cure Paralysis, University of Miami, Miami, Florida.
} 
at reducing the initial degree of neurologic injury and improving patient functional capacity are lacking (Hawryluk et al., 2008; Rowland et al., 2008).

There is a pressing unmet need to accurately prognosticate, early after SCI, patient functional outcome. During the first few days after SCI, definitive management strategies are formulated, which often include aggressive surgical decompression of the spinal cord. This is also the time of greatest anguish for an injured patient and their family as they face significant prognostic uncertainty. From a research perspective, it is difficult to meaningfully analyze the efficacy of novel interventions without defining homogenous subgroups.

Unfortunately, physicians and researchers have little to scientifically guide their prediction of outcome following SCI. The few existing studies often employ variables collected outside of the initial injury period (more than 3 days post$\mathrm{SCI}$ ), and are therefore less useful as acute clinical prediction tools (Van Middendorp et al., 2011; Zorner et al., 2010).

To improve the ability of clinicians to predict long-term outcome in the acute clinical setting, and to aid in the classification of patients within clinical trials, we aimed to create a clinical prediction model that relates clinical and imaging findings to functional outcome at 1 year following SCI.

\section{Methods}

\section{Data source}

Our analyses are based on individual patient data from the combination of two prospective datasets: the North American Clinical Trials Network for SCI (NACTN) database, and the Surgical Timing in Acute Spinal Cord Injury Study (STASCIS) database. NACTN is a prospective registry that has been enrolling patients with acute traumatic SCI from nine North American Centers since 2006. STASCIS is a prospective, multicenter, cohort study investigating the role of surgical timing in SCI (Fehlings et al., 2012). This study involved hospitals at six institutions throughout North America during a 7-year enrollment period between August 2002 and September 2009. The NACTN and STASCIS datasets contain similar acute data elements, as well as long-term functional and neurological outcome data at 6- and 12-month follow-up points. Patients with severe head injury were not enrolled in either database. After research ethics board approval at each participating site was obtained, the datasets were harmonized based on their common data elements to produce a single database.

For all patients, a neurologic examination was performed at patient presentation in accordance with the recommendations of the American Spinal Injury Association (ASIA) by a trained physician, nurse, or research assistant (American Spinal Injury Association, 2000). Injury characteristics were then classified according to neurologic level of injury (NLI), ASIA motor score (AMS), ASIA sensory score (ASS) and the overall ASIA Impairment Scale (AIS) grade. To identify neuroanatomical injury characteristics, patients underwent magnetic resonance imaging (MRI) of the spine shortly after hospital presentation. All patients received appropriate medical support, which included permissive or induced hypertensive therapy (mean blood pressure $>85 \mathrm{~mm} \mathrm{Hg}$; Hadley et al., 2002a). Methylprednisolone was used at the discretion of the treating team, according to the recommendations of the
NASCIS-2 study, and based on established guidelines (Bracken et al., 1990; Hadley et al., 2002b). Decisions surrounding surgery, including the timing of decompression, were made by the attending spine surgeon in each case. All patients underwent an individualized rehabilitation protocol, tailored to specific patient needs and injury characteristics.

Using the combined dataset, the cohort of interest was defined to include patients 16 years of age and older, presenting to an acute care facility with traumatic SCI and neurological deficit (AIS grade A-D). Only patients with a documented ASIA neurological examination performed within 3 days of injury, and with a functional independence measure (FIM) motor score available at 6 months or 1 year follow-up, were included. The former criterion was included to ensure that only acute variables were used as predictors in the development of the prediction model.

\section{Predictor variables}

Clinical and radiological predictors were chosen based on three main criteria: (1) author consensus of predictive importance of a given variable; (2) literature support for the predictive importance of a given variable; and (3) the ease with which the given variable could be collected, interpreted, and recorded, within the acute injury setting. Based on these criteria, four variables were selected to form the basis of model construction: (1) acute AIS grade, (2) acute ASIA motor score (dichotomized at a score of 50), (3) patient age at injury, and (4) intramedullary signal characteristics on the spinal MRI (Table 1A). All four of these predictors have consistently demonstrated prognostic significance in relation to long-term functional outcome after SCI (Burns et al., 1997; Cifu et al., 1999; Crozier et al., 1992; Furlan and Fehlings, 2009; Kay et al., 2007; Liao et al., 2005; Marino and Graves, 2004; Shimada and Tokioka, 1999; Tewari et al., 2005). The two examinationrelated predictor variables, AIS grade and AMS, were assessed and recorded within 3 days of injury for all patients. If an individual patient underwent more than one neurological examination within the first 3 days, we utilized the first examination that took place at acute hospital admission. Spinal MRI was also performed within 3 days of injury in all cases, and read by a site-specific neuroradiologist. Standard protocols for MR image acquisition were used, with a twodimensional spin echo sequence used for acquiring the T1 images, and a two-dimensional fast-recovery fast spin-echo sequence used for acquiring the T2 images. Age was not categorized, but rather its full variability was preserved in the form of a continuous predictor, as is the recommended practice (Harrell, 2001; Steyerberg, 2009).

\section{Outcome and follow-up}

The primary outcome variable was FIM motor score at 1 year post-injury (Table 1B). The FIM motor score consists of 13 items that assess functioning across four different domains that include self-care, sphincter control, transfers, and locomotion. The performance level for each item is strictly defined and ranges in value from 1-7, where 1 indicates complete dependence in an activity, and a score of 6 or greater indicates that a patient is capable of performing that activity independently, without supervision or help. The result is a discrete outcome variable with a minimum value of 13 and a maximum value of 91, with a larger value implying superior 
Table 1. Predictor and Outcome Variables

Predictor Coding

A. Pre-specified predictor variables

Initial ASIA Impairment Scale (AIS) grade

Initial ASIA Motor Score (AMS)

Age

Spinal magnetic resonance imaging intramedullary signal characteristics

B. Functional independence motor (FIM) motor score
1. Self-care
A. Eating /7
B. Grooming /7
C. Bathing /7
D. Dressing-upper body /7
E. Dressing-lower body $/ 7$
F. Toileting / 7
2. Sphincter control
G. Bladder management /7
$\mathrm{H}$. Bowel management $/ 7$
3. Transfers
I. Bed, chair, wheelchair /7
J. Toilet /7
K. Tub, shower $/ 7$
4. Locomotion
L. Walk/wheelchair /7
M. Stair /7
Total score

ASIA, American Spinal Injury Association.

functional status. This outcome measure has been shown to be valid and reliable for use in the setting of SCI (Dodds et al., 1993; Hamilton et al., 1994). If outcome assessment was not available at 1 year, the 6-month FIM motor score was used, a practice that has been used and shown to be valid in previous studies (Van Middendorp et al., 2009,2011). As a secondary outcome variable, we dichotomized the primary outcome, identifying all of the patients who achieved a score of at least 6 for all of the FIM motor score items. We considered these patients to have achieved functional independence across the 13 items assessed, as has been the practice in previous strokerelated research (Stineman et al., 1997).

\section{Statistical methods}

Model creation. All analyses were performed using SAS version 9.3 (SAS Institute, Cary, NC), and R statistical software version 2.14.1. Multi-colinearity was assessed through multivariate linear regression variable tolerance testing, with a variable tolerance threshold set at 0.4 . To account for missing data among the predictors, a multiple imputations procedure with 10 imputation iterations was performed using Markov chain Monte Carlo methods. This resulted in an augmented dataset containing 10 times the original sample size, all with complete data. Such imputation is recommended as being less susceptible to bias and more efficient than performing a complete case analysis by dropping cases with incomplete variables (Harrell, 2001; Little, 1992). Using the imputed dataset, with follow-up FIM motor score as the dependent variable, and initial AIS grade, dichotomized AMS, age, and intramedullary MRI signal characteristics as the prespecified four covariates, a linear regression model was generated for each of the 10 imputation iterations. A multiple imputation analysis procedure was then employed, which combines the results of the analyses for each of the imputation iterations to provide a single set of parameter estimates. Stepwise statistical variable selection procedures such as forward inclusion or backward elimination were not employed, as such techniques have been shown to result in biased estimates of regression coefficients and exaggeration of variable $p$ values (Harrell, 2001; Murray et al., 2007; Steyerberg, 2009). R-squared values from each of the imputation iterations were averaged to quantify the overall model performance.

A binary logistic regression model was created with the dependent variable of functional independence modeled 
Table 2. Patient Characteristics at Hospital Admission

\begin{tabular}{|c|c|c|c|c|}
\hline Characteristic & $\begin{array}{l}\text { Patients meeting } \\
\text { inclusion/ } \\
\text { exclusion criteria } \\
\mathrm{n}=696\end{array}$ & $\begin{array}{l}\text { Cohort } \\
\text { without } \\
\text { follow-up } \\
\mathrm{n}=320\end{array}$ & $\begin{array}{l}\text { Cohort with } \\
\text { follow-up used } \\
\text { in final analysis } \\
\mathrm{n}=376\end{array}$ & p Value ${ }^{*}$ \\
\hline Mean age (years) & $44.5( \pm 17.3)$ & $46.1( \pm 17.5)$ & $43.2( \pm 16.9)$ & 0.03 \\
\hline Male gender & $531(76.3 \%)$ & $237(74.1 \%)$ & $294(78.2 \%)$ & $>0.05$ \\
\hline $\begin{array}{l}\text { Initial ASIA Impairment Scale (AIS) grade: } \\
\text { AIS grade A } \\
\text { AIS grade B } \\
\text { AIS grade C } \\
\text { AIS grade D }\end{array}$ & $\begin{array}{l}258(37.1 \%) \\
115(16.5 \%) \\
119(17.1 \%) \\
204(29.3 \%)\end{array}$ & $\begin{array}{r}122(38.1 \%) \\
52(16.3 \%) \\
61(19.1 \%) \\
85(26.6 \%)\end{array}$ & $\begin{array}{r}136(36.2 \%) \\
63(16.8 \%) \\
58(15.4 \%) \\
119(31.7 \%)\end{array}$ & $>0.05$ \\
\hline Initial ASIA motor score $>50$ : & $217(31.2 \%)$ & $93(29.3 \%)$ & $124(32.9 \%)$ & $>0.05$ \\
\hline $\begin{array}{l}\text { MRI intramedullary signal characteristics: } \\
\text { No signal change } \\
\text { Consistent with edema } \\
\text { Consistent with hemorrhage }\end{array}$ & $\begin{array}{r}43(14.8 \%) \\
149(51.6 \%) \\
97(33.6 \%)\end{array}$ & $\begin{array}{l}19(15.1 \%) \\
65(51.6 \%) \\
42(33.3 \%)\end{array}$ & $\begin{array}{l}24(14.7 \%) \\
84(51.5 \%) \\
55(33.7 \%)\end{array}$ & $>0.05$ \\
\hline $\begin{array}{l}\text { Etiology: } \\
\text { Motor vehicle accident } \\
\text { Fall } \\
\text { Sport or diving accident } \\
\text { Other }\end{array}$ & $\begin{array}{r}283(40.7 \%) \\
247(35.5 \%) \\
70(10.1 \%) \\
96(13.8 \%)\end{array}$ & $\begin{array}{c}137(42.8 \%) \\
115(35.9 \%) \\
24(7.5 \%) \\
44(13.8 \%)\end{array}$ & $\begin{array}{r}146(38.8 \%) \\
132(35.1 \%) \\
46(12.2 \%) \\
52(13.8 \%)\end{array}$ & $>0.05$ \\
\hline Received steroids & $410(60.7 \%)$ & $183(59.0 \%)$ & $227(62.1 \%)$ & $>0.05$ \\
\hline $\begin{array}{l}\text { Mean time to decompressive surgery } \\
\text { post-injury (hours) }\end{array}$ & $75.3( \pm 429.4)$ & $74.5( \pm 429.4)$ & $76.1( \pm 338.9)$ & $>0.05$ \\
\hline
\end{tabular}

*For the difference between the group with and that without follow-up.

ASIA, American Spinal Injury Association; MRI, magnetic resonance imaging.

relative to the same four predictor variables. The model was tested through the construction of receiver operator characteristic (ROC) curves, for each of the imputation iterations. The area under the curve (AUC) is a summary measure of the discriminative ability of the model, with values between 0.90 and 1.00 indicative of excellent predictive discrimination. AUC values from each of the imputation iterations were averaged to create a single value quantifying model discrimination. Lastly, to evaluate the added predicted value of the full four-predictor model over AIS grade alone, a likelihood ratio (LR) test was used to compare the LR chi-square value from the full logistic model, to the LR chi-square value of a model containing AIS grade as a lone predictor. A $p$ value for this test $<0.05$ would indicate that the predictive capacity of the full model is significantly larger than the predictive capacity of AIS grade used as a lone predictor.

Model validation. Internal validation of the linear and logistic prediction models was performed using a bootstrap re-sampling procedure of the imputed dataset using 200 individual bootstrap replicates (each with $n=376$ ). The prespecified models were fitted to each, and a $95 \%$ confidence interval $(95 \% \mathrm{CI})$ was generated about the mean of the 200 replicate parameter estimate values for each covariate. The mean R-squared and AUC values across the bootstrap replicates were also generated and were assumed to be an unbiased estimate of the true values.

Finally, the regression coefficients obtained from the internally-validated linear and logistic models were used to estimate FIM motor score and the probability of achieving functional independence at 1 year follow-up for hypothetical
SCI patients based on their unique clinical and radiologic characteristics at hospital admission.

\section{Results \\ Study population}

Prospective data from the NACTN and STASCIS protocols were combined to produce a single harmonized dataset of 729 patients. From this original cohort, 33 patients did not have a neurological assessment performed within the first 3 days after injury and were excluded. Demographic, injury, and imaging characteristics for the remaining 696 patients are shown in Table 2.

\section{Functional outcomes}

FIM motor score evaluations were available in 310 patients at 1 year, and in 66 patients at 6 months, for a total of 376 patients on which to base the analysis (Fig. 1). There were no significant differences in initial AIS grade, AMS, or MRI intramedullary signal characteristics, between patients included and those excluded due to inadequate follow-up ( $p>0.05$; Table 2). Of the patients included in the analysis, 227 (62.1\%) received steroids, and $358(96.5 \%)$ underwent decompressive surgery. There were no significant differences in the proportion of steroid administration or in the mean time to surgery between patients included and those excluded due to inadequate follow-up ( $p>0.05 p$; Table 2$)$. The mean FIM motor score at follow-up was $62.9( \pm 28.6)$, with incrementally higher mean scores seen with a progressively less severe acute admission AIS grade (Supplementary Table S1; see online 


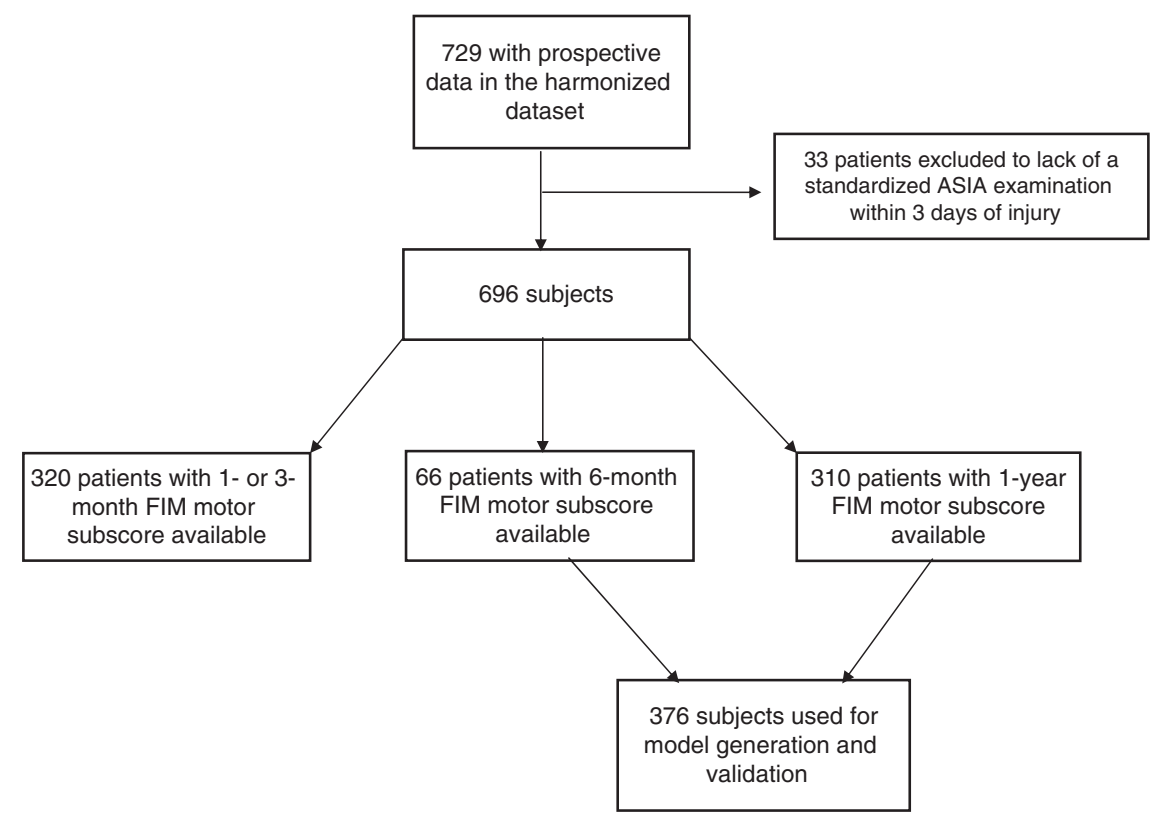

FIG. 1. Flow chart of the study design.

supplementary material at http://www.liebertonline.com). One-hundred and forty-eight patients (39.4\%) achieved functional independence (a score of at least 6 on all of the FIM motor score items), while 228 patients (60.6\%) failed to achieve this milestone. Information on neurological recovery is available in Supplementary Table S2 (see online supplementary material at http://www.liebertonline.com).

\section{Model development and validation}

Predictor variable parameter estimates, significance values, and bootstrapped estimates are shown in Table 3A for the linear model, and 3B for the logistic model. For both models, the mean parameter estimate values for each covariate across the 200 bootstrap replicates closely approximated the estimates from the original dataset. Furthermore, the 95\% confidence intervals around these mean values are narrow, and contain the parameter estimates generated from the original dataset. For the linear model, performance, judged by the Rsquared values averaged across each of the 10 imputation iterations, was 0.52 (minimum 0.51, maximum 0.54). The mean R-squared value across the bootstrap replicates was 0.52 (95\% CI 0.52,0.53), which is identical to the model performance in the original dataset. For the logistic model based on the dichotomized outcome variable, the AUC value averaged across the 10 imputation iterations was 0.93 (minimum 0.91 ,

Table 3. Parameter Estimates for Models Predicting (A) Fim Motor Score, and (B) Functional Independence, at 1-Year Follow-Up from Original Sample and Bootstrap Replicates

\begin{tabular}{|c|c|c|c|c|}
\hline Prognostic variable & Parameter estimate & & p Value & $\begin{array}{l}\text { Bootstrap parameter } \\
\text { estimate }(95 \% \mathrm{CI})\end{array}$ \\
\hline \multicolumn{5}{|l|}{ A. } \\
\hline Intercept & 50.28 & & $<0.01$ & $49.73(49.0,50.5)$ \\
\hline Age & -0.33 & & $<0.01$ & $-0.33(-0.34,-0.32)$ \\
\hline Admission AMS > 50 & 9.17 & & $<0.01$ & $9.11(8.61,9.61)$ \\
\hline Admission AIS grade & 12.47 & & $<0.01$ & $12.54(12.34,12.76)$ \\
\hline MRI signal & -4.83 & & 0.19 & $-4.65(-4.91,-4.40)$ \\
\hline Prognostic variable & Parameter estimate & Odds ratio & p Value & $\begin{array}{c}\text { Bootstrap parameter } \\
\text { estimate }(95 \% \mathrm{CI})\end{array}$ \\
\hline \multicolumn{5}{|l|}{ B. } \\
\hline Intercept & -2.93 & - & $<0.01$ & $-2.99(-3.11,-2.87)$ \\
\hline Age & -0.03 & 0.97 & $<0.01$ & $-0.03(-0.03,-0.03)$ \\
\hline Admission AMS > 50 & 1.35 & 3.86 & $<0.01$ & $1.34(1.27,1.41)$ \\
\hline Admission AIS grade & 1.36 & 3.90 & $<0.01$ & $1.39(1.27,1.41)$ \\
\hline MRI signal & -0.29 & 0.75 & 0.54 & $-0.30(-0.34,-0.25)$ \\
\hline
\end{tabular}

ASIA, American Spinal Injury Association; AMS, ASIA motor score; MRI, magnetic resonance imaging; 95\% CI, 95\% confidence interval; AIS, ASIA Impairment Scale; FIM, functional independence measure. 
FIM motor 1-year $=50.28-0.33($ Age $)+9.17($ AMS $)+12.47($ AIS grade $)-4.83($ MRI signal $)$

Prob. Independence 1-year $=\exp [-2.93-0.03($ Age $)+1.35($ AMS $)+1.36($ AIS grade $)-0.29($ MRI Signal $)]$ $(1+\exp [-2.93-0.03($ Age $)+1.35($ AMS $)+1.36($ AIS grade $)-0.29$ (MRI Signal) $]$

Where :

Age: continuous variable $>16$

AMS: ASIA Motor Score $<$ or $=50$; ASIA Motor Score $>50=1$;

AIS grade: AIS grade $A=1$; AIS grade $B=2$; AIS grade $C=3$; AIS grade $D=4$;

MRI Signal: No signal change=0; Sig. cons. w/edema=1; Sig. cons. w/hemor=2;

EXP: Natural logarithm

FIG. 2. Predictive model equations (ASIA, American Spinal Injury Association; AMS, ASIA motor score; MRI, magnetic resonance imaging; FIM, functional independence measure; AIS, ASIA Impairment Scale).

maximum 0.93), indicating excellent model predictive discrimination. The mean AUC value across the bootstrap replicates was 0.92 (95\% CI 0.92,0.93). The likelihood ratio test comparing the full four-predictor logistic model to a model containing AIS grade as a lone predictor revealed a $p$ value $<0.01$, based on a chi-square statistic of 23.5 and three degrees of freedom. Figure 2 presents the internally-validated prediction model equations.

Lastly, using the regression coefficients from the internallyvalidated models, we estimated the FIM motor score and the probability of achieving functional independence at 1 year follow-up for a variety of hypothetical SCI patient scenarios (Table 4).

\section{Discussion}

We have produced the first prediction models using acute clinical and radiologic data, obtained within the first three days after injury, to predict long term functional outcome after traumatic spinal cord injury. Four pre-specified predictor variables, ASIA Impairment Scale, dichotomized ASIA motor score, age and MRI intra-medullary signal characteristics, were modeled relative to Functional Independence Measure motor score for a large prospective cohort of SCI patients. The results of this modeling indicate that better functional status is predicted by a sequentially less severe initial AIS grade and an AMS greater than 50 at hospital admission. In contrast worse functional outcomes are predicted by older age and by MRI intra-medullary signal characteristics consistent with spinal cord edema or hemorrhage. The parameter estimates generated for these predictors were validated using a bootstrap resampling procedure of observations from the original dataset. This research has generated a simple equation that can be used to estimate long-term functional outcome by substituting the applicable variables into the equation for each patient at acute hospital admission. Next, by dichotomizing the primary outcome measure and generating a secondary logistic model, the same four predictor variables demonstrated an excellent ability to discriminate an individual who will achieve functional independence, from one who will not achieve this threshold. Lastly, by performing a likelihood ratio test we demonstrated that the full logistic model displayed significantly greater

Table 4. Predicted Estimates for FiM Motor Score and the Probability of Achieving Functional Independence at 1-Year Post-Injury for a Hypothetical Set of SCI Patients

\begin{tabular}{|c|c|c|c|c|c|c|}
\hline Characteristic & Patient 1 & Patient 2 & Patient 3 & Patient 4 & Patient 5 & Patient 6 \\
\hline Age (years) & 75 & 75 & 55 & 55 & 25 & 25 \\
\hline AIS A & Yes & No & Yes & No & No & No \\
\hline AIS B & No & No & No & No & No & Yes \\
\hline AIS C & No & No & No & No & Yes & No \\
\hline AIS D & No & Yes & No & Yes & No & No \\
\hline AMS > 50 & No & Yes & No & Yes & Yes & No \\
\hline MRI hemorrhage & Yes & No & Yes & No & No & No \\
\hline MRI edema & No & No & No & No & Yes & Yes \\
\hline Predicted FIM motor score & 28 & 85 & 44 & 91 & 84 & 62 \\
\hline Probability of functional independence & 0.01 & 0.83 & 0.08 & 0.90 & 0.72 & 0.22 \\
\hline
\end{tabular}

ASIA, American Spinal Injury Association; AMS, ASIA motor score; MRI, magnetic resonance imaging; AIS, ASIA Impairment Scale; FIM, functional independence measure. 
predictive capacity than a logistic model containing AIS grade as a lone predictor.

A number of individual clinical, radiographic and demographic factors have been shown to be of importance in predicting clinical outcome after traumatic SCI(Browne, B., et al., 1993, Burns, A. and Ditunno, J. 2001, Calancie, B., et al., 2004, Coleman, W. and Geisler, F. 2004, Crozier, K., et al., 1991, Furlan, J. and Fehlings, M. 2009, Lazar, R., et al., 1989, Pollard, M. and Apple, D. 2003). However, studies that assess the significance of combining individual parameters to improve outcome predictions are sparse in the literature. Zoerner et al produced a clinical algorithm to predict ambulatory capacity six months post SCI, based on 90 patients with incomplete SCI (79\% traumatic, 21\% non-traumatic)(Zorner, B., et al., 2010). A more recent study from the European Multicenter SCI study group generated a clinical prediction rule for future ambulatory outcomes using age, motor scores from the quadriceps and soleus muscles and light touch sensations in the L3 an S1 dermatomes as predictor variables(Van Middendorp, J., et al., 2011). This analysis was based on traumatic SCI patients with neurological examination variables obtained up to 15 days after injury, with the vast majority of examinations taking place three days after injury.

These studies provide evidence that use of a combination of variables may enable a more reliable prediction of long-term outcome after SCI as compared to the use of single predictors in isolation. However, a number of key limitations in the existing literature must be recognized. All of the prognostic studies employing a combination of variables have relied largely on measurements obtained outside the critical first three days after the injury: the period during which the majority of acute therapeutic decisions are made and a time during which there is significant demand for the delivery of accurate prognostic information. Previous studies have demonstrated that extraneous patient and injury related variables in the acute setting can interfere with the accuracy of the initial neurological examination, yielding results that are discordant with those obtained later post admission (Cadotte, D., et al., 2011, Maynard, F., et al., 1979). Hence, previous prediction rules formed using data outside the acute period, are less suitable for use at the time of patient admission to hospital, a fact which diminishes their clinical and research utility. Given our reliance on acute care data obtained exclusively within three days of injury for all patients, the current prognostic model can be used at the time of admission by acute care practitioners to estimate longterm prognosis.

Many previous studies exploring the predictors of future functional status have utilized walking as the primary measure of long-term outcome(Burns, S., et al., 1997, Calancie, B., et al., 2004, Crozier, K., et al., 1992, Van Middendorp, J., et al., 2011). While future ambulatory capacity is of interest, it is of paramount importance to consider other dimensions of functional status. In a large survey assessing quality of life in over 200,000 SCI patients, regaining arm and hand function was the highest priority for quadriplegics, while regaining bowel/bladder and sexual function were the highest priorities for paraplegics(Anderson, K. 2004). Moreover, only a small fraction of patients recover ambulatory capacity after SCI, with a maximum of $5 \%$ of patients with complete SCI becoming ambulatory at one year, irrespective of the initial neurological level of injury(Waters, R., et al., 1993, Waters, R., et al., 1992). These results underscore the importance of incorporating outcome tools that include multi-dimensional assessments of functional status. In the current study we utilized the FIM motor score which, in addition to mobility status, assesses items related to self-care, sphincter control and transfers, areas of functioning that have great relevance to the majority of SCI patients on a daily basis. One potential drawback of utilizing this outcome measure is its lack of interpretability: for a given patient with a specific predicted value, it is difficult to precisely articulate exact functional expectations within each of the four outcome domains based on the cumulative score. We have attempted to mitigate this concern by producing a second model which predicts functional independence, a more interpretable recovery threshold based on dichotomized FIM motor score.

\section{Study Limitations}

While applying the pre-specified models to the bootstrap replicates confirms their validity within our own dataset, establishing true external validity of this prediction rule will require an evaluation of its performance within a separate group of SCI patients. Before such an evaluation takes place, the generalizability of this model, as well as its suitability for use in the clinical realm, remains unknown. We also recognize that a large number of patients failed to be included in the analysis due to inadequate follow-up. However, we have demonstrated that, with the exception of age, no significant differences existed in the baseline characteristics between those included in the analysis and those excluded due to inadequate follow-up. The difference in age (46.1 in patients with missing follow-up and 43.2 in patients with follow-up), although statistically significant, is not significant from a clinical standpoint. We also recognize that treatment related factors not included in our model, such as time to decompressive surgery or steroid administration, may have influenced patient outcomes in this study. However this model was intended to incorporate only non-treatment predictors related to the natural history of recovery for SCI patients. Lastly, while a standardized MRI protocol was used for the acute evaluation of patients within this study, we acknowledge the existence of other MR sequences, such as gradient echo, that may have provided a more sensitive measure of intramedullary hemorrhage.

\section{Conclusion}

Given the clinical and pathologic heterogeneity of traumatic SCI, identifying a single variable to account for the full spectrum of patient recovery is a formidable challenge. In response to this, we have created, and internally validated, a prediction model for functional outcome at 1 year, based on ASIA Impairment Scale grade, dichotomized ASIA motor score, age, and MRI intramedullary signal characteristics, with all predictor variables obtained within the first 3 days after injury for all patients. After establishing the performance of this model within a separate cohort of patients, we look forward to its implementation as a prognostic tool in the realm of acute clinical care, as well as a stratification tool in future clinical trials to help produce balanced treatment groups. As additional future steps, we also look forward to investigating the predictive capacity of different imaging modalities and MR imaging sequences, as well as to 
producing models specifically focused on predicting outcome for important SCI patient subgroups, such as those with incomplete injuries or central cord syndrome.

\section{Acknowledgments}

The authors wish to acknowledge the support of the following agencies and granting bodies who contributed to the study: The Christopher and Dana Reeve Foundation, the Cervical Spine Research Society, and the Rick Hansen Institute. The authors also thank Elizabeth Toups and Yuriy Petrenko for logistical support, and Keith Burau as well as Ting Ou for assistance with database management. The authors thank Michele M. Johnson of the University of Texas Health Sciences Centre for her contributions to the article.

\section{Author Disclosure Statement}

No competing financial interests exist.

\section{References}

American Spinal Injury Association. (2000). International Standards for Neurological Classification of Spinal Cord Injury. ASIA: Chicago.

Anderson, K. (2004). Targeting recovery: priorities of the spinal cord injured population. J. Neurotrauma 10, 1371-1383.

Bracken, M., Shepard, M., Collins, W., Holford, T., Young, W., Baskin, D., Eisenberg, H., Flamm, E., Leo-Summers, L., Maroon, J., and Marshall, L. (1990). A randomized, controlled trial of methylprednisolone or naloxone in the treatment of acute spinal-cord injury. Results of the Second National Acute Spinal Cord Injury Study. N. Engl. J. Med. 17, 1405-1411.

Browne, B., Jacobs, S., Herbison, G., and Ditunno, J. (1993). Pin sensation as a predictor of extensor carpi radialis recovery in spinal cord injury. Arch. Phys. Med. Rehab. 74, 14-18.

Burns, A., and Ditunno, J. (2001). Establishing prognosis and maximizing functional outcomes after spinal cord injury. Spine 26, S137-S145.

Burns, S., Golding, D., Rolle, W., Graziani, V., and Dittuno, J. (1997). Recovery of ambulation in motor-incomplete tetraplegia. Arch. Phys. Med. Rehab. 78, 1169-1172.

Cadotte, D., Wilson, J., Mikulis, D., Stroman, P., Brady, S., and Fehlings, M. (2011). Conventional MRI as a diagnostic and prognostic tool in spinal cord injury. Expert Opin. Med. Diag. 5, 1-13.

Calancie, B., Molano, M., and Broton, J. (2004). Tendon reflexes for prediction movement recovery after acute spinal cord injury in humans. Clin. Neurophysiol. 115, 2350-2363.

Christopher and Dana Reeve Foundation. (2011). Paralysis Facts and Figures.

Cifu, D., Seel, R., Kreutzer, J., and McKinley, W. (1999). A multicenter investigation of age-related differences in lengths of stay, hospitalization charges, and outcomes for a matched tetraplegic sample. Arch. Phys. Med. Rehab. 80, 733-740.

Coleman, W., and Geisler, F. (2004). Injury severity as primary predictor of outcome in acute spinal cord injury: retrospective results from a large multicenter clinical trial. Spine J. 4, 373-378.

Crozier, K., Cheng, L., Graziani, V., Zorn, G., Herbison, G., and Ditunno, J. (1992). Spinal cord injury: prognosis for ambulation based on quadriceps recovery. Paraplegia 30, 762-767.

Crozier, K., Graziani, V., Dittuno, J., and Herbison, G. (1991). Spinal cord injury: Prognosis for ambulation based on sensory examination in patients who are initially motor complete. Arch. Phys. Med. Rehab. 72, 118-121S.
Dodds, T., Martin, D., Stolov, W., and Devo, R. (1993). A validation of the functional independence measurement and its performance among rehabilitation inpatients. Arch. Phys. Med. Rehab. 74, 531-536.

Fehlings, M.G., Wilson, J., Vaccaro, A., Singh, A., Cadotte, D., Harrop, J., Aarabi, B., Shaffrey, C.I., Dvorak, M., Fisher, C., Arnold, P., Massicotte, E., Lewis, S., and Rampersaud, R. (2012). Early versus delayed decompression for traumatic cervical spinal cord injury: Results of the Surgical Timing in Acute Spinal Cord Injury Study (STASCIS). PLoS One 7, 1-8.

Furlan, J., and Fehlings, M. (2009). The impact of age on mortality, impairment, and disability among adults with acute traumatic spinal cord injury. J. Neurotrauma 26, 1707-1717.

Hadley, M., Walters, B., Grabb, P., Oyesiku, N., Przybylski, G., Resnick, D., and Ryken, T. (2002a). Blood pressure management after acute spinal cord injury. Neurosurgery 50, S58-S62.

Hadley, M., Walters, B., Grabb, P., Oyesiku, N., Przybylski, G., Resnick, D., and Ryken, T. (2002b). Pharmacological therapy after acute cervical spinal cord injury. Neurosurgery 50, 563-572.

Hamilton, B., Laughlin, J., Fiedler, R., and Granger, C. (1994). Interrater reliability of the 7-level functional independence measure (FIM). Scand. J. Rehab. Med. 26, 115-119.

Harrell, F. (ed.) (2001). Regression Modeling Strategies with Applications to Linear Models, Logistic Regression and Survival Analysis. Springer-Verlag: New York.

Hawryluk, G., Roland, J., Kwon, B., and Fehlings, M. (2008). Protection and repair of the injured spinal cord: a review of completed, ongoing, and planned clinical trials for acute spinal cord injury. Neurosurgical Focus 25, E14.

Kay, E., Deutsch, A., and Wuermser, L. (2007). Predicting walking at discharge from inpatient rehabilitation after a traumatic spinal cord injury. Arch. Phys. Med. Rehab. 88, 745750.

Lazar, R., Yarkony, G., Ortolano, D., Heinemann, A., Perlow, E., and Lovell, L. (1989). Prediction of functional outcome by motor capacity after spinal cord injury. Arch. Phys. Med. Rehab. 70, 819-822.

Liao, C., Lui, T., Chen, L., Chuang, C., and Huang, Y. (2005). Spinal cord injury without radiological abnormality in preschool-aged children: correlation of magnetic resonance imaging findings with neurological outcomes. J. Neurosurgery 103, S17-S23.

Little R. (1992). Regression with missing X's: a review. J Am Stat Assoc. 88:125-134.

Marino, R., and Graves, D. (2004). Metric properties of the ASIA motor score: Subscales improve correlation with functional activities. Arch. Phys. Med. Rehab. 85, 1804-1810.

Maynard, F., Reynolds, G., Fountain, S., Wilmot, C., and Hamilton, R. (1979). Neurologic prognosis after traumatic quadriplegia. J. Neurosurg. 50, 611-616.

Murray, G., Butcher, I., McHugh, G., Lu, J., Mushkudiani, N., Maas, A., Marmarou, A., and Steyerberg, E. (2007). Multivariate prognostic analysis in traumatic brain injury: Results from the Impact Study. J Neurotrauma 24, 329-337.

Pollard, M., and Apple, D. (2003). Factors associated with improved neurologic outcomes in patients with incomplete tetraplegia. Spine 28, 33-39.

Rowland, J., Hawryluk, G., Kwon, B., and Fehlings, M. (2008). Current status of acute spinal cord injury pathophysiology and emerging therapies: promise on the horizon. Neurosurgical Focus 25, E2.

Shimada, K., and Tokioka, T. (1999). Sequential MR studies of cervical cord injury: correlation with neurological damage and clinical outcome. Spinal Cord 37, 410-415. 
Steyerberg, E. (ed.) (2009). Clinical Prediction Models: A Practical Approach to Development, Validation, and Updating. Springer: New York.

Stineman, M., Maislin, G., Fiedler, R., and Granger, C. (1997). A prediction model for functional recovery in stroke. Stroke 28, 550-556.

Tewari, M., Gifti, D., and Singh, P. (2005). Diagnosis and prognostication of adult spinal cord injury without radiographic abnormality using magnetic resonance imaging: analysis of 40 patients. Surg. Neurol. 63, 204-209.

Van Middendorp, J., Hosman, A., Donders, A., Pouw, M., Dittuno, J., Curt, A., Geurts, A., and Van de Meent, H. (2011). A clinical prediction rule for ambulation outcomes after traumatic spinal cord injury: a longitudinal cohort study. Lancet 377, 1004-1010. van Middendorp, J., Hosman, A., Pouw, M., and Van de Meent, $\mathrm{H}$. (2009). Is determination between complete and incomplete traumatic spinal cord injury clinically relevant? Validation of the ASIA sacral sparing criteria in a prospective cohort of 432 patients. Spinal Cord 47, 809-816.

Waters, R., Adkins, R., Yakura, J., and Sie, I. (1993). Motor and sensory recovery following complete tetraplegia. Arch. Phys. Med. Rehab. 74, 242-247.
Waters, R., Yakura, J., Adkins, R., and Sie, I. (1992). Recovery following complete paraplegia. Arch. Phys. Med. Rehab. 73, 784-789.

Wyndaele, M., and Wyndaele, J. (2006). Incidence, prevalence, and epidemiology of spinal cord injury: what learns a worldwide literature survey? Spinal Cord 44, 523-529.

Zorner, B., Blanckenhom, W., Dietz, V., and Group E-SS. (2010). Clinical algorithm for improved prediction of ambulation and patient stratification after incomplete spinal cord injury. J. Neurotrauma 27, 241-252.

Address correspondence to: Michael G. Fehlings, M.D., Ph.D., FRCSC, FACS Department of Neurosurgery University of Toronto Head Spinal Program 399 Bathurst Street Toronto Western Hospital Toronto, Ontario, Canada M5T 2S8

E-mail: Michael.Fehlings@uhn.on.ca 